\title{
Mechanical Behavior of Fiber-to-concrete Interface in Textile Reinforced Concrete: Theoretical Model
}

\author{
Shanshan Cheng \\ School of Engineering, University of Plymouth, Plymouth, Devon PL4 8AA, UK \\ E-mail: shanshan.cheng@plymouth.ac.uk
}

Received: 12 September 2018; Accepted: 26 September 2018; Available online: 1 March 2019

\begin{abstract}
This paper presents a theoretical solution of a reinforcement-to-concrete interface model under pullpush loading. Expressions for the interfacial shear stress distribution and load-displacement history are derived for different loading stages. The full debonding propagation process is discussed in detail and the analytical solutions are verified by comparing with existing theoretical models. Results of the analytical solution are presented to illustrate how the bond length and local bond-slip law affect the interfacial bond behavior. While the case study in this paper is on textile reinforced concrete, the analytical solution is equally valid to similar mechanical cases such as rebar reinforced concretes.
\end{abstract}

Keywords: Bond-slip; Interface; Analytical solution; Textile reinforced concrete.

\section{Introduction}

In recent years, fiber-reinforced polymer (FRP) materials have been widely used in reinforced concrete in place of conventional steel reinforcement thanks to their advantages such as high strength, light weight and great corrosion resistance in marine environment. Conventionally FRP has been produced in bar shapes to imitate the steel rebar. However, the application has been restricted due to difficulties in bending the FRP bars at the connections. In contrast, textile fabrics made of fiber bundles have well offset those limitations. Therefore, textile reinforced concrete has been increasingly used recently since they enable the production of lightweight and aesthetically appealing members. To understand the structural behavior of the textile reinforced concrete, the bond between the textile and the concrete has been a key concern. To experimentally investigate the bond performance, the quasi-standard pullout test has been widely used [1]. While a great deal of research is now available on the mechanical behavior of the bonded interface, no closed form analytical solution has been presented which is capable of predicting the entire debonding propagation. This paper is to present such an analytical solution using a trilinear local bond-slip law.

\section{1D analytical model}

A double-sided pullout test setup, originally developed at the RWTH Aachen University in Germany, has been illustrated in Figure 1. The test follows the concept of the conventional steel rebar reinforced concrete, where the reinforcement is loaded under tension, while concrete is under compression.

Figure 2 shows a schematic diagram of the pull-push test of a singular textile roving reinforced concrete model, where the cross-sectional area of the three components (reinforcement, adhesive layer and concrete) are constant along the length. The width and thickness of the concrete are denoted by $b_{c}$ and $t_{c}$ respectively. The textile reinforcement is assumed to be homogenous with a circular cross-section (diameter of $\varnothing$ ), where the unloaded transverse rovings of the mesh are excluded. The bonded length is denoted by $L$. The Young's modulus of the reinforcement and the concrete are $E_{p}$ and $E_{c}$ respectively. A horizontal coordinate system originating from the left end is adopted (Figure 2). In such a model, the adhesive layer is mainly subjected to shear deformations, so mode II interfacial fracture is the dominate failure mode. The concrete and the reinforcement are assumed to be subjected to axial deformations only. Note that in such a model, the adhesive layer represents not only the deformation of the actual adhesive layer, but also that of a thin layer of the adjacent concrete and is referred to in this paper as the interface. 


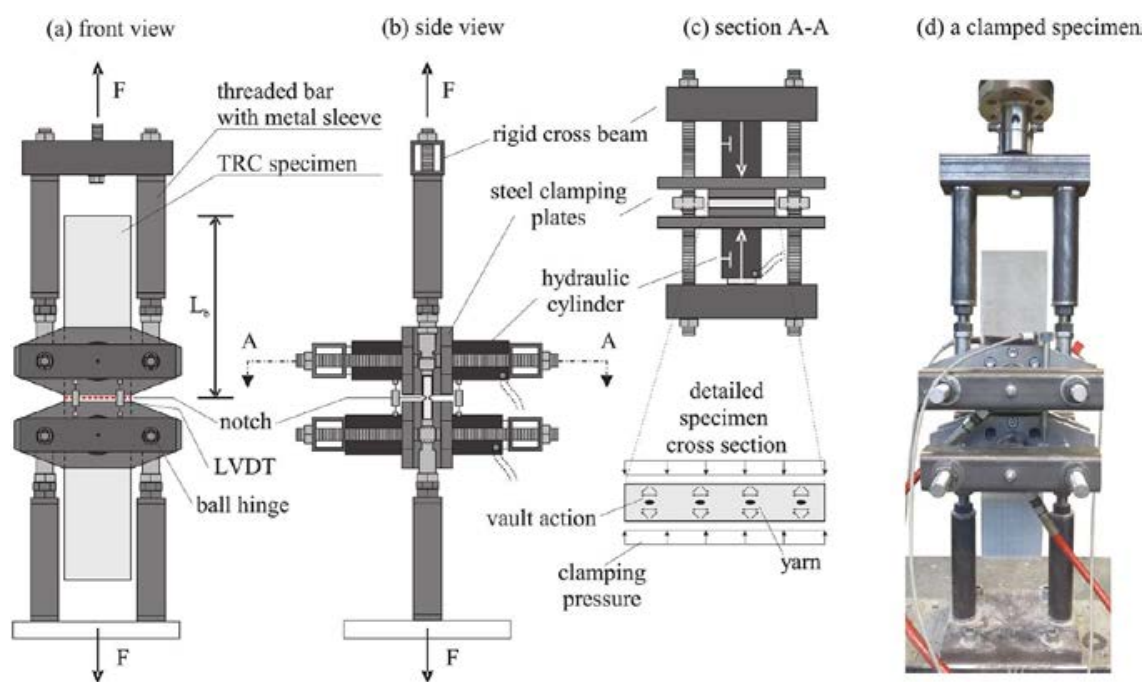

Figure 1 Pullout test setup for textile reinforced concrete [1]

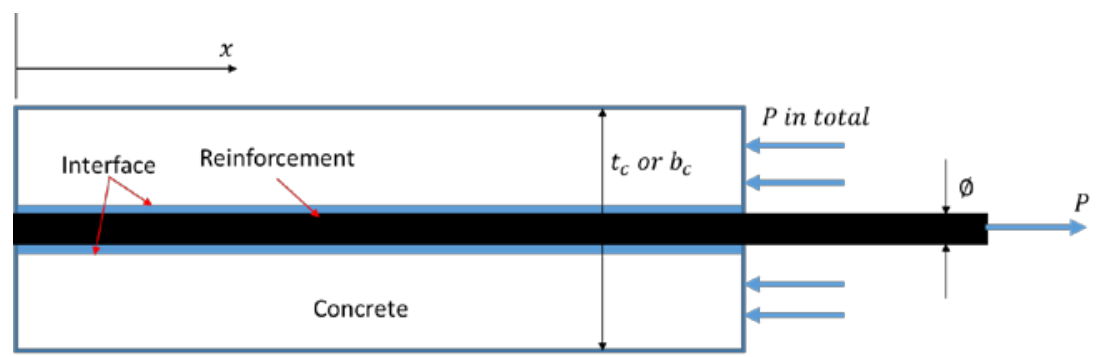

Figure 2 Idealized push-pull model of reinforcement-to-concrete bonded interface

The differential equation expressing equilibrium conditions along the reinforcement can be defined as

$$
\begin{aligned}
& A_{p} \frac{d \sigma_{p}}{d x}-\pi \emptyset \tau=0 \\
& A_{p} \sigma_{p}+A_{c} \sigma_{c}=0
\end{aligned}
$$

where $A_{p}=\frac{\pi \phi^{2}}{4}$ is the cross-sectional area of the reinforcement, and $A_{c}=b_{c} t_{c}$ is the cross-sectional area of the concrete; $\sigma_{p}$ and $\sigma_{c}$ are the axial stress in the reinforcement and the concrete respectively, and $\tau$ is the bond stress. Assuming both the reinforcement and the concrete are in the elastic range during the bond test, the constitutive equations of the adhesive layer and the two adherends are to Eqs. (3-5)

$$
\begin{aligned}
& \tau=f(\delta) \\
& \sigma_{p}=E_{p} \frac{d u_{p}}{d x} \\
& \sigma_{c}=E_{c} \frac{d u_{c}}{d x}
\end{aligned}
$$

The interfacial slip $\delta$ is defined as the relative displacement between the two adherends:

$\delta=u_{p}-u_{c}$

After substituting Eqs. (2-6) into Eq. (1), the governing equation is obtained:

$\frac{d^{2} \delta}{d x^{2}}-\lambda^{2} \tau=0$

where $\lambda^{2}=\pi \emptyset \gamma$ and $\gamma=\frac{1}{E_{p} A_{p}}+\frac{1}{E_{c} A_{c}}$.

The axial stresses in the reinforcement and concrete could be reformatted as follows: 


$$
\begin{aligned}
\sigma_{p} & =\frac{1}{A_{p} \gamma} \frac{d \delta}{d x} \\
\sigma_{c} & =-\frac{\sigma_{p} A_{p}}{A_{c}}
\end{aligned}
$$

Once a local bond-slip relationship $f(\delta)$ is given, a pullout test can be simulated and compared with the experimental data. Various local bond-slip law has been developed in the past decades, for example, the wellrecognized modified Bertero-Eligehausen-Popov (mBEP) model and the Cosenza-Manfredi-Realfonzo (CMR) model $[2,3]$. However, the above equilibrium equations cannot be solved in a closed form using those bond-slip models, and thereby computing software are necessary to simulate the interface behavior. In order to obtain a closed form solution, which offers unique advantages in parametric studies and design, the trilinear or bilinear (special case of a trilinear model) bond-slip laws are more favorable. This paper adopts a trilinear bond-slip law (as shown in Eq. (10) and Figure 3). The bond shear stress increases linearly with the interfacial slip until it reaches the peak stress $\tau_{f}$ at which the value of the slip is denoted by $\delta_{1}$. Interfacial softening (or micro-cracking) then starts with the shear stress reducing linearly with the increase of the interfacial slip. The shear fracture (or debonding) occurs when the interfacial slip reaches $\delta_{2}$. The residual shear stress $\tau_{r}$ implies the friction and aggregate interlock over the debonded length. If the aggregate interlock is ignored, $\tau_{r}=0$ and it yields a bilinear local bond-slip law.

$$
f(\delta)=\left\{\begin{array}{lr}
\frac{\tau_{f}}{\delta_{1}} \delta & 0 \leq \delta \leq \delta_{1} \\
-\frac{\tau_{f}-\tau_{r}}{\delta_{2}-\delta_{1}} \delta+\frac{\tau_{f} \delta_{2}-\tau_{r} \delta_{1}}{\delta_{2}-\delta_{1}} & \delta_{1} \leq \delta \leq \delta_{2} \\
\tau_{r} & \delta \geq \delta_{2}
\end{array}\right.
$$

Using the bond-slip model defined in Eq. (10), the governing equation (7) can be solved to find the shear stress distribution along the interface and the load-displacement response of the specimen. Figure 4 shows the interfacial shear stress distribution and the propagation of debonding. Note that the interfacial shear stress distribution only holds when the bond length is substantially higher than the critical softening length $a_{u}$ for the transfer of the ultimate load (Eq. 46).

\subsection{Elastic stage}

At the beginning of loading, the entire length of the interface is in an elastic stress state (state I, Figure 4(a)), until the interfacial shear stress at $x=L$ reaches $\tau_{f}$. Substituting Eq. (10) into Eq. (7), the following differential equation is obtained:

$$
\frac{d^{2} \delta}{d x^{2}}-\lambda_{1}^{2} \delta=0
$$

where $\lambda_{1}{ }^{2}=\lambda^{2} \frac{\tau_{f}}{\delta_{1}}$

Using the boundary conditions of

$$
\sigma_{p}=0 \text { at } x=0 ; \quad \sigma_{p}=\frac{P}{A_{p}} \text { at } x=L
$$

The governing equation (11) could be solved:

$$
\delta=\frac{\delta_{1}}{\tau_{f}} \frac{P \lambda_{1} \cosh \left(\lambda_{1} x\right)}{\pi \phi \sinh \left(\lambda_{1} L\right)}
$$

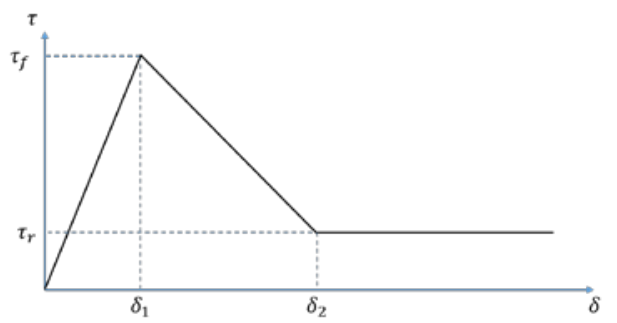

Figure 3 Local bond-slip law 


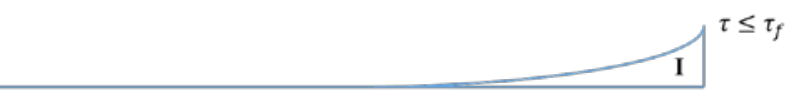

(a) Elastic stress state

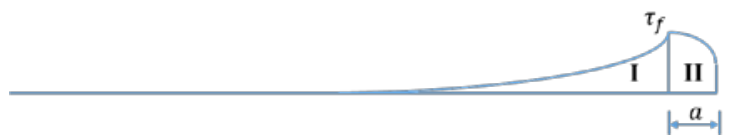

(b) Propagation of softening zone

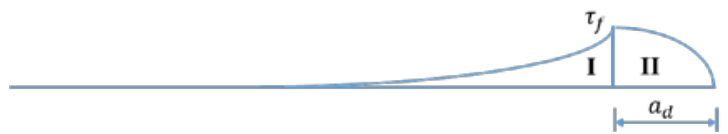

(c) Initiation of debonding at $\mathrm{x}=\mathrm{L}$

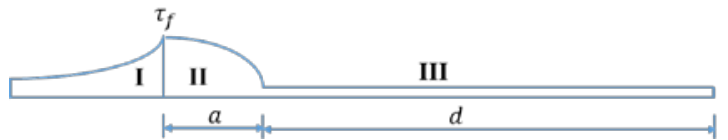

(d) Propagation of debonding

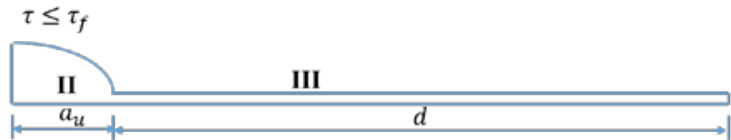

(e) Unloading

Figure 4 Interfacial shear stress distribution and propagation of debonding for a large bond length

and thus the interfacial shear stress and the axial stress in the reinforcement are:

$$
\begin{gathered}
\tau=\frac{P \lambda_{1} \cosh \left(\lambda_{1} x\right)}{\pi \phi \sinh \left(\lambda_{1} L\right)} \\
\sigma_{p}=\frac{P \sinh \left(\lambda_{1} x\right)}{A_{p} \sinh \left(\lambda_{1} L\right)}
\end{gathered}
$$

Eqs. (14-16) are identical to Yuan et al. [4] by replacing the contact width $b_{p}$ with the contact perimeter $\pi \phi$. Thus the equation of the effective bond length (defined as the bond length over which the shear stresses offer a total resistance of at least $97 \%$ of the applied load) in the paper [4] still holds:

$$
l_{e, e}=\frac{2}{\lambda_{1}}
$$

From Eq. (14), it is found that the relative slip at the loaded end $(x=L)$ is:

$$
\Delta=\frac{\delta_{1}}{\tau_{f}} \frac{P \lambda_{1}}{\pi \phi} \operatorname{coth}\left(\lambda_{1} L\right)
$$

and thus the load-displacement relationship could be written as:

$$
\mathrm{P}=\frac{\tau_{f} \pi \phi \Delta}{\lambda_{1} \delta_{1}} \tanh \left(\lambda_{1} L\right)
$$

Note that the elastically bonded stage of the interface ends when the relative slip at the loaded end reaches $\delta_{1}$. Therefore the load at the elastic limit is

$$
\mathrm{P}=\frac{\tau_{f} \pi \phi}{\lambda_{1}} \tanh \left(\lambda_{1} L\right)
$$

\subsection{Elastic-softening stage}

Once the load $P$ has exceeded the elastic limit, the softening occurs at the loaded end of the interface (state II, Figure 4(b)). Part of the interface enters softening state (state II) while the rest remains elastic (state I). The load $P$ continues to increase as the length of the softening zone $a$ increases. Substituting the bond-slip relationship into Eq. (7) gives the following governing equations: 


$$
\begin{array}{ll}
\frac{d^{2} \delta}{d x^{2}}-\lambda_{1}{ }^{2} \delta=0 & 0 \leq \delta \leq \delta_{1} \\
\frac{d^{2} \delta}{d x^{2}}+\lambda_{2}{ }^{2} \delta=\lambda_{2}{ }^{2} e & \delta_{1} \leq \delta \leq \delta_{2}
\end{array}
$$

where

$$
\lambda_{2}^{2}=\lambda^{2} \frac{\tau_{f}-\tau_{r}}{\delta_{2}-\delta_{1}}
$$

and

$$
e=\frac{\tau_{f} \delta_{2}-\tau_{r} \delta_{1}}{\tau_{f}-\tau_{r}}
$$

The boundary conditions at this stage are:

$\sigma_{p}=0$ at $x=0$;

$\sigma_{p}$ is continuous at $x=L-a$;

$\delta=\delta_{1}$ or $\tau=\tau_{f}$ at $x=L-a ;$

$\sigma_{p}=\frac{P}{A_{p}}$ at $x=L$

The solution for the elastic region of the interface $\left(0 \leq \delta \leq \delta_{1}\right.$ or $\left.0 \leq x \leq L-a\right)$ is given by:

$\delta=\delta_{1} \frac{\cosh \left(\lambda_{1} x\right)}{\cosh \left[\lambda_{1}(L-a)\right]}$

and thus the interfacial shear stress and the axial stress in the reinforcement are:

$\tau=\tau_{f} \frac{\cosh \left(\lambda_{1} x\right)}{\cosh \left[\lambda_{1}(L-a)\right]}$
$\sigma_{p}=\frac{\pi \phi \delta_{1} \lambda_{1}}{A_{p} \lambda^{2}} \frac{\sinh \left(\lambda_{1} x\right)}{\cosh \left[\lambda_{1}(L-a)\right]}$

The solution for the softening region of the interface $\left(\delta_{1} \leq \delta \leq \delta_{2}\right.$ or $\left.L-a \leq x \leq L\right)$ is given by:

$$
\begin{aligned}
& \delta=\left(\delta_{1}-e\right) * \cos \left[\lambda_{2}(L-a-x)\right]-\delta_{1} \frac{\lambda_{1}}{\lambda_{2}} \tanh \left[\lambda_{1}(L-a)\right] * \sin \left[\lambda_{2}(L-a-x)\right]+e \\
& \tau=\tau_{f} * \cos \left[\lambda_{2}(L-a-x)\right]+\delta_{1} \frac{\lambda_{1}}{\lambda_{2}} \frac{\tau_{f}-\tau_{r}}{\delta_{2}-\delta_{1}} \tanh \left[\lambda_{1}(L-a)\right] * \sin \left[\lambda_{2}(L-a-x)\right] \\
& \sigma_{p}=\frac{\pi \phi}{A_{p} \lambda^{2}}\left\{\left(\delta_{1}-e\right) * \lambda_{2} * \sin \left[\lambda_{2}(L-a-x)\right]+\delta_{1} \lambda_{1} \tanh \left[\lambda_{1}(L-a)\right] * \cos \left[\lambda_{2}(L-a-x)\right]\right\}
\end{aligned}
$$

Thus the relative slip at the loaded end $(x=L)$ is

$$
\Delta=\left(\delta_{1}-e\right) * \cos \left(\lambda_{2} a\right)+\delta_{1} \frac{\lambda_{1}}{\lambda_{2}} \tanh \left[\lambda_{1}(L-a)\right] * \sin \left(\lambda_{2} a\right)+e
$$

Combining Eqs. (28) and (34) yields

$$
P=\frac{\pi \phi}{\lambda^{2}}\left\{-\left(\delta_{1}-e\right) * \lambda_{2} * \sin \left(\lambda_{2} a\right)+\delta_{1} \lambda_{1} \tanh \left[\lambda_{1}(L-a)\right] * \cos \left(\lambda_{2} a\right)\right\}
$$

If $a=0$, Eq. (36) returns to Eq. (20). If $\tau_{r}=0$, the governing equations and solutions are identical to the equations in Yuan et al. [4]. Therefore the derivation in this paper is valid. 
When the relative slip at the loaded end $\Delta$ reaches $\delta_{2}$, the elastic-softening stage finishes, and debonding commences and propagates along the interface. The corresponding value of $a$, denoted by $a_{d}$ can be obtained from Eq. (35) as

$$
\left(\delta_{1}-e\right) * \cos \left(\lambda_{2} a_{d}\right)+\delta_{1} \frac{\lambda_{1}}{\lambda_{2}} \tanh \left[\lambda_{1}\left(L-a_{d}\right)\right] * \sin \left(\lambda_{2} a_{d}\right)+e-\Delta=0
$$

\subsection{Elastic-softening-debonding stage}

During this stage of loading, debonding commences and propagates along the interface. The interfacial shear stress distribution along the interface is shown in Figure 4(d). As debonding propagates, the peak shear stress $\tau_{f}$ moves towards $x=0$. Assuming that the debonded length of the interface starting at the loaded end is $d$, Eqs. (2934) are still valid if replacing $L$ by $(L-d)$. Therefore, in the region of $0 \leq \delta \leq \delta_{1}$ or $0 \leq x \leq L-d-a$ :

$$
\delta=\delta_{1} \frac{\cosh \left(\lambda_{1} x\right)}{\cosh \left[\lambda_{1}(L-d-a)\right]}
$$

and thus the interfacial shear stress and the axial stress in the reinforcement are:

$$
\begin{aligned}
& \tau=\tau_{f} \frac{\cosh \left(\lambda_{1} x\right)}{\cosh \left[\lambda_{1}(L-d-a)\right]} \\
& \sigma_{p}=\frac{\pi \phi \delta_{1} \lambda_{1}}{A_{p} \lambda^{2}} \frac{\sinh \left(\lambda_{1} x\right)}{\cosh \left[\lambda_{1}(L-d-a)\right]}
\end{aligned}
$$

In the region of $\delta_{1} \leq \delta \leq \delta_{2}$ or $L-d-a \leq x \leq L-d$

$$
\begin{aligned}
& \delta=\left(\delta_{1}-e\right) * \cos \left[\lambda_{2}(L-d-a-x)\right]-\delta_{1} \frac{\lambda_{1}}{\lambda_{2}} \tanh \left[\lambda_{1}(L-d-a)\right] * \sin \left[\lambda_{2}(L-d-a-x)\right]+e \\
& \tau=\tau_{f} * \cos \left[\lambda_{2}(L-d-a-x)\right]+\delta_{1} \frac{\lambda_{1}}{\lambda_{2}} \frac{\tau_{f}-\tau_{r}}{\delta_{2}-\delta_{1}} \tanh \left[\lambda_{1}(L-d-a)\right] * \sin \left[\lambda_{2}(L-d-a-x)\right] \\
& \sigma_{p}=\frac{\pi \phi}{A_{p} \lambda^{2}}\left\{\left(\delta_{1}-e\right) * \lambda_{2} * \sin \left[\lambda_{2}(L-d-a-x)\right]+\delta_{1} \lambda_{1} \tanh \left[\lambda_{1}(L-d-a)\right] * \cos \left[\lambda_{2}(L-d-a-x)\right]\right\}
\end{aligned}
$$

The elastic interface disappears when the relative slip at the unloaded end $(x=0)$ is $\delta_{1}$. Using Eq. (38), it is found that the elastic stage finishes when

$$
L-d=a
$$

Using the boundary condition of $\delta=\delta_{2}$ at $x=L-d$, the relationship between $d$ and $a$ is obtained by using Eq. (41):

$$
\delta_{2}=\left(\delta_{1}-e\right) * \cos \left(\lambda_{2} a\right)+\delta_{1} \frac{\lambda_{1}}{\lambda_{2}} \tanh \left[\lambda_{1}(L-d-a)\right] * \sin \left(\lambda_{2} a\right)+e
$$

Combining Eqs. (44) and (45), the softening length $a_{u}$ when the elastic interface disappears is obtained as:

$$
a_{u}=\frac{\operatorname{arcos}\left(\frac{\delta_{2}-e}{\delta_{1}-e}\right)}{\lambda_{2}}
$$

In the debonded region, that is $\delta \geq \delta_{2}$ or $L-d \leq x \leq L$, the governing equation is:

$$
\frac{d^{2} \delta}{d x^{2}}-\lambda^{2} \tau_{r}=0
$$

Using the continuous boundary conditions at $x=L-d$, the solution is found as:

$$
\begin{aligned}
& \delta=\frac{\lambda^{2} \tau_{r}}{2}\left[x^{2}-(L-d)^{2}\right]+\delta_{2}+(L-d-x)\left(\delta_{1}-e\right) \lambda_{2} \sin \left(\lambda_{2} a\right)-(L-d-x) \delta_{1} \lambda_{1} \tanh \left[\lambda_{1}(L-d-\right. \\
& a)] \cos \left(\lambda_{2} a\right)+\lambda^{2} \tau_{r}(L-d)(L-d-x)
\end{aligned}
$$


Thus the relative slip at the loaded end $(\mathrm{x}=\mathrm{L})$ is:

$\Delta=\frac{\lambda^{2} \tau_{r}}{2} d^{2}+\delta_{2}-d\left(\delta_{1}-e\right) \lambda_{2} \sin \left(\lambda_{2} a\right)+d \delta_{1} \lambda_{1} \tanh \left[\lambda_{1}(L-d-a)\right] \cos \left(\lambda_{2} a\right)$

In the debonded region, the interfacial shear stress is $\tau_{r}$. Thus the load $P$ could be obtained by

$P=P(x=L-d)+\tau_{r} \pi \phi d$

$=\frac{\pi \phi}{\lambda^{2}}\left\{-\left(\delta_{1}-e\right) * \lambda_{2} * \sin \left(\lambda_{2} a\right)+\delta_{1} \lambda_{1} \tanh \left[\lambda_{1}(L-d-a)\right] * \cos \left(\lambda_{2} a\right)\right\}+\tau_{r} \pi \phi d$

Eq. (50) is consistent with Yuan, et.al [4] if the residual shear stress $\tau_{r}=0$.

\subsection{Softening-debonding stage}

This stage is governed by Eqs. (22) and (47), with the following boundary conditions:

$\sigma_{p}=0$ at $x=0$;

$\sigma_{p}$ is continuous at $x=a$;

$\delta=\delta_{2}$ or $\tau=\tau_{f}$ at $x=a ;$

$\sigma_{p}=\frac{P}{A_{p}}$ at $x=L$, or $P_{(x=L)}=P_{(x=a)}+\tau_{r} \pi \phi$

In the softening range $(0 \leq x \leq a)$, the solutions of the governing equations are obtained as:

$$
\begin{aligned}
& \delta=\frac{\delta_{2}-e}{\cos \left(\lambda_{2} a\right)} \cos \left(\lambda_{2} x\right)+e \\
& \tau=-\frac{\tau_{f}-\tau_{r}}{\delta_{2}-\delta_{1}} \frac{\delta_{2}-e}{\cos \left(\lambda_{2} a\right)} \cos \left(\lambda_{2} x\right)-\frac{\tau_{f}-\tau_{r}}{\delta_{2}-\delta_{1}} e+\frac{\tau_{f} \delta_{2}-\tau_{r} \delta_{1}}{\delta_{2}-\delta_{1}} \\
& \sigma_{p}=-\frac{\pi \phi \lambda_{2}}{A_{p} \lambda^{2}} \frac{\delta_{2}-e}{\cos \left(\lambda_{2} a\right)} \sin \left(\lambda_{2} x\right)
\end{aligned}
$$

In the debonded range, the solutions are:

$$
\begin{aligned}
& \delta=\frac{\lambda^{2} \tau_{r}}{2}(x-a)^{2}+\delta_{2}-\lambda_{2}\left(\delta_{2}-e\right) \tan \left(\lambda_{2} a\right)(x-a) \\
& \sigma_{p}=-\frac{\pi \phi}{A_{p} \lambda^{2}}\left[\lambda^{2} \tau_{r}(x-a)-\lambda_{2}\left(\delta_{2}-e\right) \tan \left(\lambda_{2} a\right)\right]
\end{aligned}
$$

Thus the relative slip at the loaded end $(x=L)$ is

$$
\Delta=\frac{\lambda^{2} \tau_{r}}{2}(L-a)^{2}+\delta_{2}-\lambda_{2}\left(\delta_{2}-e\right) \tan \left(\lambda_{2} a\right)(L-a)
$$

and the load $P$ is:

$$
P=-\frac{\pi \phi \lambda_{2}}{\lambda^{2}}\left(\delta_{2}-e\right) \tan \left(\lambda_{2} a\right)+\tau_{r} \pi \phi d
$$

\section{Parametric studies}

The material properties of the carbon fiber textile reinforcement given in paper [5] are adopted in this study. The Young's modulus of the carbon fiber is 230GPa, and the roving cross-sectional area (24k filament) is 0.889 $\mathrm{mm}^{2}$, which results in a diameter of $1.1 \mathrm{~mm}$. C100 concrete are used for parametric studies, where the Young's modulus of the concrete varies between is taken as $45 \mathrm{GPa}$. Textile mesh size between $5 \mathrm{~mm}$ and $25 \mathrm{~mm}$ are commonly used in practice, which represents a concrete width of $5 \mathrm{~mm}$ to $25 \mathrm{~mm}$ for each roving. The thickness of concrete between textile layers is usually between $5 \mathrm{~mm}$ and $20 \mathrm{~mm}$, which will be adopted in the parametric studies in this paper. The local bond-slip law is estimated using paper [5], where $\delta_{1}=0.01 \mathrm{~mm}, \delta_{2}=0.7 \mathrm{~mm}, \tau_{f}=$ 7.2MPa, and $\tau_{r}=2 \mathrm{MPa}$. The mechanical behavior of the fiber-concrete interface will be discussed in this section. 


\subsection{Load-displacement curve}

Figure 5 shows the load-displacement curve of the pull-push test of a textile reinforced concrete. If not specified, the cross-sectional area of the concrete is taken as $80 \mathrm{~mm}^{2}$, and the local bond-slip law is taken as $\delta_{1}=$ $0.01 \mathrm{~mm}, \delta_{2}=0.7 \mathrm{~mm}, \tau_{f}=7.2 \mathrm{MPa}$, and $\tau_{r}=2 \mathrm{MPa}$. As the analytical solution does not represent the unloading range (softening-debonding stage) reasonably, only Figure 5(a) has included them for illustration purposes. It is seen that the bond length does not affect the load-displacement behavior obviously. Longer bond length may provide better ductility owing to its high deformation capacity. However bear in mind that the analytical solution is based on the assumptions of elastic material properties. Therefore when the bond length is very long, debond may not be the critical failure mode compared with material yielding or fracture. The interfacial residual shear stress $\tau_{r}$ has a significant effect on the hardening behavior. Thus the mechanical friction caused by aggregate interlocking plays a significant role in bond performance. Surface treatment of carbon fiber textiles could be an effective solution in improving mechanical behavior of textile reinforced concrete. Same effect has been seen on the ultimate relative slip $\delta_{2}$, which also affect the hardening behavior of the textile reinforced concrete considerably. Compared with $\delta_{2}, \delta_{1}$ only affects the bond behavior insignificantly.

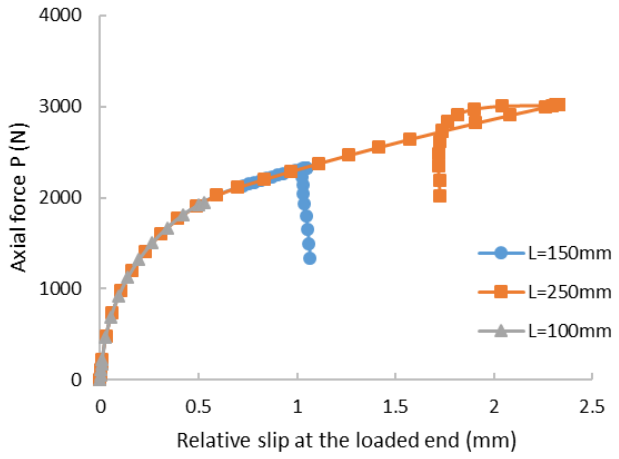

(a) Effect of bond length

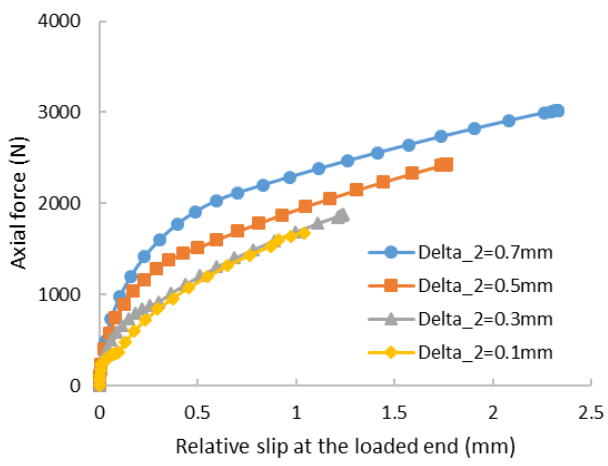

(c) Effect of $\delta_{2}$

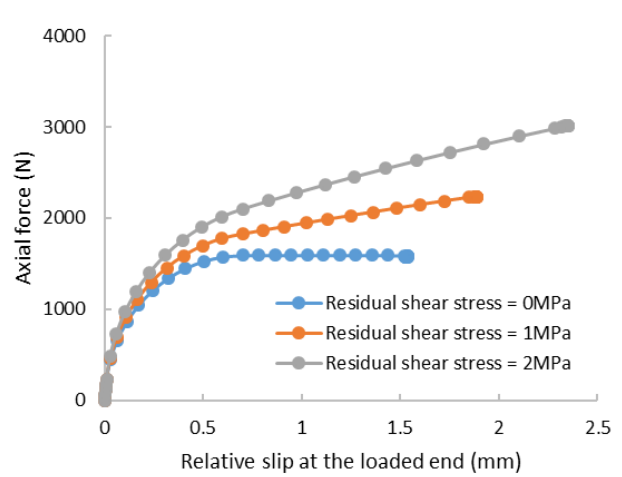

(b) Effect of interfacial residual shear stress $\tau_{r}$

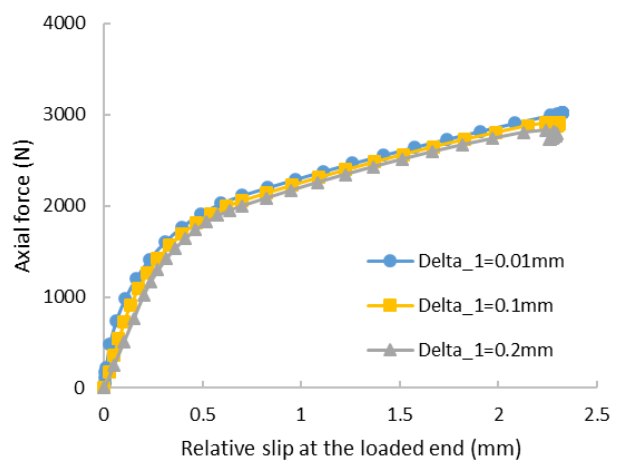

(d) Effect of $\delta_{1}$

Figure 5 Load displacement curves of pull-push bond specimens

\subsection{Stress distributions}

Figure 6 demonstrates the stress distributions along the interface of the specimen. A bond length of $250 \mathrm{~mm}$ and a cross-sectional area of $50 \mathrm{~mm}^{2}$ have been chosen for this case study. The interfacial shear stress distribution has agreed well with the diagram sketched in Figure 4. Note that the tensile strength of carbon fiber is commonly seen between 3GPa and 4GPa. The carbon fiber would have snapped in the softening-debonding stage in this case. Therefore a specimen in practice will not be able to reach the ultimate debonding force prediction in this paper, which is to say the analytical solution derived in this paper provides an upper bound of the interfacial bond behavior.

\section{Conclusions}

This paper has presented a closed-form solution of the pull-push model of a textile reinforced concrete. The analytical solution enables the prediction of the full debonding propagation process, including the interfacial shear stress distributions and axial stresses in the reinforcement and the concrete. This will facilitate users to understand the mechanical behavior of textile-to-concrete interface and to optimize the bond requirement and reinforcement volume in concrete. Note that the analytical solution is based on the assumption that the bond length is sufficiently 
long so that the ultimate load could be effectively transferred. If the bond length is smaller than the critical softening distance $a_{u}$ (Eq. (46)), the interface may not experience all stages as shown in Figure 4, and therefore the load-displacement history may vary. This will be discussed in future work.

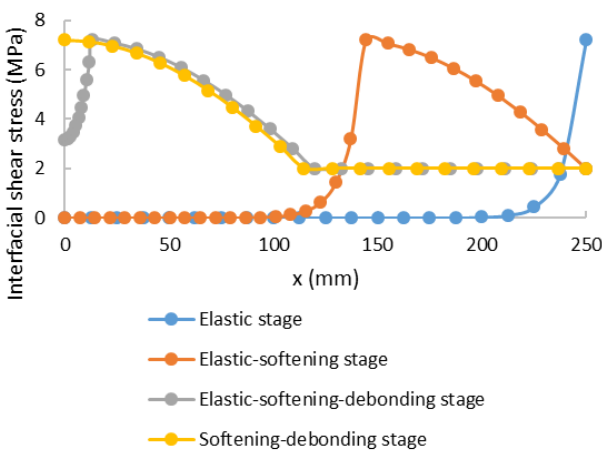

(a) Interfacial shear stress distribution

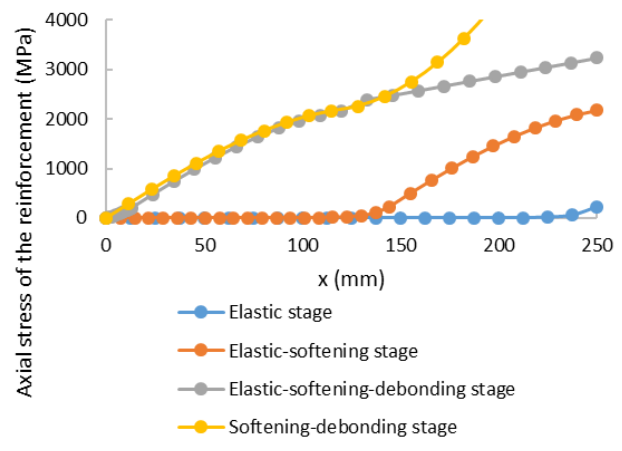

(b) Axial stress distribution of the reinforcement

Figure 6 Local stress distributions along the specimen

\section{References}

[1] Li Y, Bielak J, Hegger J, Chudoba R. An incremental inverse analysis procedure for identification of bondslip laws in composites applied to textile reinforced concrete. Composites Part B: Engineering. 2018;137: 111-122.

[2] Focacci F, Nanni A, Bakis CE. Local bond-slip relationship for FRP reinforcement in concrete. ASCE Journal of Composites for Construction. 2000;4(1):24-31.

[3] Lin X, Zhang YX. Evaluation of bond stress-slip models for FRP reinforcing bars in concrete. Composite Structures. 2014; 107:131-141.

[4] Yuan H, Teng JG, Seracino R, Wu ZS, Yao J. Full-range behavior of FRP-to-concrete bonded joints. Engineering Structures. 2004;26(5): 553-565.

[5] Portal NW, Perez IF, Thrane LN, Lundgren K. Pull-out of textile reinforcement in concrete. Construction and Building Materials. 2014;71: 63-71.

(C) 2019 by the author(s). This work is licensed under a Creative Commons Attribution 4.0 International License (http://creativecommons.org/licenses/by/4.0/). Authors retain copyright of their work, with first publication rights granted to Tech Reviews Ltd. 